\title{
Conhecimento da equipe de enfermagem acerca da Prevenção de infecção em sítio cirúrgico
}

\author{
Knowledge of the nursing team about the prevention of surgical site infection \\ Conocimiento del equipo de enfermería sobre la prevención de infección en sitio \\ quirúrgico
}

João Victor Borges Veras Cronemberger ${ }^{1 *}$, Saraí de Brito Cardoso ${ }^{1}$, Maria Zélia de Araújo Madeira ${ }^{1}$, Ivonizete Pires Ribeiro ${ }^{1}$, Mariana de Fátima Barbosa de Alencar ${ }^{1}$.

\section{RESUMO}

Objetivo: Analisar o conhecimento da equipe de enfermagem do centro cirúrgico sobre as medidas de prevenção de infecção em sítio cirúrgico. Métodos: Trata-se de uma pesquisa exploratória e descritiva, com abordagem quantitativa, desenvolvida em um hospital privado localizado, em Teresina, Piauí. A amostra da pesquisa foi constituída pelos profissionais de Enfermagem: 03 enfermeiros e 23 técnicos de Enfermagem. Resultados: Os dados foram analisados através das frequências absolutas (№) e relativas (\%). Foi possível evidenciar que os profissionais de enfermagem, objeto da amostra, demonstraram conhecimento satisfatório sobre a prevenção de infecção no sítio cirúrgico, visto que obtiveram resultados de $80 \%$ a $100 \%$ de acertos na maior parte do questionário. Conclusão: Há necessidade de estratégias de aperfeiçoamento e atualização no que diz respeito a prevenção de infecção de sítio cirúrgico.

Palavras-chave: Infecção da ferida cirúrgica, Equipe de Enfermagem, Medidas preventivas.

\begin{abstract}
Objective: To analyze the knowledge of the nursing team of the surgical center about the measures of prevention of infection in a surgical site. Methods: This is an exploratory and descriptive study, with a quantitative approach, developed in a private hospital located in Teresina, Piauí. The research sample consisted of Nursing professionals: 03 nurses and 23 Nursing technicians. Results: The data were analyzed through the absolute (No.) and relative (\%) frequencies. It was possible to show that the nursing professionals, the sample of the sample, showed satisfactory knowledge about the prevention of infection in the surgical site, since they obtained results of $80 \%$ to $100 \%$ of correct answers in most of the questionnaire. Conclusion: There is a need for improvement and update strategies regarding the prevention of surgical site infection.
\end{abstract}

Keywords: Surgical wound infection, Nursing team, Preventive measures.

\section{RESUMEN}

Objetivo: Análisis del equipo de enfermería y del centro cirúrgico sobre las medidas de prevención de la información en el campo. Métodos: Trata-se de uma pesquisa exploratória and descritiva, com abordagag quantitativa, desenvolvida en un hospital privado localizado, en Teresina, Piauí. A amostra da pesquisa foi constituída pelos profissionais de Enfermagem: 03 enfermeiros e 23 técnicas de Enfermagem. Resultados: Datos dados por análisis analógico de frecuencias absolutas (№) y relativas (\%). Además, se ha comprobado que las profesiones son de enfermería, objetos de trabajo, demostraciones de contacto satisfactorios sobre la prevención de la información en el ámbito cirúrgico, y hemos obtenido resultados del $80 \%$ de los $100 \%$ de los defectos en el trabajo. Conclusión: La necesidad de estrategias de apertura y de atención al cliente no tiene que ver con una prevención de la información del estudio.

Palabras clave: Infección de la herida quirúrgica, Equipo de Enfermería, Medidas preventivas. INTRODUÇÃO

${ }^{1}$ Centro Universitário Uninovafapi, Teresina-PI. *E-mail: jcronemberger@outlook.com 
A infecção relacionada à Assistência à Saúde (IRAS) é qualquer infecção que venha a ser adquirida após a internação hospitalar do paciente e pode se manifestar durante a internação ou mesmo após a alta do paciente - quando puder ser relacionada com a internação e/ou procedimentos invasivos (PADOVEZE MC; FORTALEZA CMCB, 2014).

A Organização Mundial de Saúde (OMS) preconiza três medidas para prevenções de infecções de sítio cirúrgico, sendo elas: a segurança do paciente, identificação de eventos adversos ocorridos e a minimização dos seus efeitos com intervenções eficazes. Trata-se de medidas de extrema importância, visto que $75 \%$ dos casos de Infecção do Sítio Cirúrgico (ISC) são diagnosticados no período posterior à alta hospitalar (ANVISA, 2013).

A Infecção do Sítio Cirúrgico (ISC) é uma das infecções pertencentes à assistência à saúde no Brasil. Ficou constatado que atualmente ela está ocupando as primeiras posições entre todos os tipos de infecções em serviços de saúde. Nos casos de pacientes hospitalizados, o índice de infecção é de $14 \%$ a $16 \%$. Ao analisar essa taxa, pode ser notada uma grande importância dos fatores relativos à população atendida $\mathrm{e}$ procedimentos praticados nos serviços de saúde (REIS RG; RODRIGUES MCS, 2017).

O risco de Infecção de Sítio Cirúrgico envolve fatores intrínsecos que estão relacionados à desnutrição, idade extrema, câncer, obesidade, dentre outros e fatores extrínsecos (relacionados à cirurgia) que podem ser: tempo de degermação cirúrgica das mãos e antebraços, hospitalização prolongada, duração da cirurgia, antissepsia da pele, antibióticos profiláticos e esterilização. Embora alguns fatores não possam ser modificados como, por exemplo, a idade do paciente, outros podem ser controlados ou eliminados por meio de um excelente processo de cuidado focado na prevenção (SOBECC, 2017).

A preocupação com a infecção no centro cirúrgico tem sido destaque desde seus tempos primordiais até a atualidade. Embora o conhecimento preventivo baseado em evidências exista, hoje com o avanço dos antibióticos, equipamentos, métodos de limpezas e esterilização mais eficazes têm diminuído consideravelmente o índice de infecções relacionadas a procedimentos cirúrgicos.

Diante disso, os objetivos desta pesquisa foram analisar o conhecimento da equipe de enfermagem do centro cirúrgico de uma instituição privada de Teresina/PI sobre as medidas de prevenção de Infecção de Sítio Cirúrgico, levantar o perfil sociodemográfico e formação acadêmica dos profissionais de enfermagem atuantes no centro cirúrgico associar o conhecimento sobre medidas de prevenção de Infecção de Sítio Cirúrgico com as recomendações da ANVISA e do Ministério da Saúde - MS.

\section{MÉTODOS}

Trata-se de uma pesquisa de campo exploratória e descritiva com abordagem quantitativa. Segundo Gil AC (2008) a pesquisa do tipo descritiva tem por objetivo descrever as características de determinadas populações ou fenômenos, em que uma de suas peculiaridades é a utilização de técnicas padronizadas de coleta de dados, como o uso do questionário, realizando uma observação sistemática.

O estudo exploratório é um tipo de pesquisa de campo, no qual se desenvolve uma investigação cujo objetivo é a formulação de questões ou de um problema, com a finalidade de familiarizar o pesquisador com um ambiente, fato ou fenômeno para modificar ou clarificar conceitos (MARCONI MA; LAKATOS EM, 2010).

A pesquisa quantitativa é um estudo estatístico que se destina a descrever as características de uma determinada situação, medindo numericamente as hipóteses levantadas a respeito de um problema de pesquisa (ZANELLA C, 2009).

Este estudo foi desenvolvido em um hospital privado localizado em Teresina, Piauí. É um hospital geral que atende alta complexidade, constituído por 100 leitos de internação, pronto atendimento geral e cardiológico, unidade de terapia intensiva com 12 leitos e um centro cirúrgico de grande porte com seis salas de cirurgia. 
A população foi constituída pelos profissionais de Enfermagem: enfermeiros e técnicos de enfermagem que trabalham no Centro Cirúrgico (CC). Esta equipe é composta por 03 enfermeiros e 23 técnicos de enfermagem. $O$ estudo foi do tipo censitário para todos os profissionais que atuam no CC.

Foram incluídos no estudo os funcionários que compõe a equipe de Enfermagem (enfermeiros e técnicos) do Centro Cirúrgico que se disponibilizaram a participar dessa coleta de dados.

Foram excluídos os enfermeiros e os técnicos de enfermagem que se encontraram de férias e os profissionais afastados para tratamento de saúde. Para a realização do estudo foi utilizado um instrumento de coleta de dados e foi aplicado no mês de agosto de 2018.

Foi feito na forma de um questionário semiestruturado, previamente testado, com questões de múltipla escolha baseadas nas recomendações de prevenção de Infecção de Sítio Cirúrgico adotados pelo Ministério da Saúde.

Os dados coletados foram digitados em um banco de dados Microsoft Office Excel que foram exportados para o programa IBM SPSS Statístics 20.0 que fez o processamento dos dados.

Os dados foram analisados de forma descritiva univariável por meio da leitura das frequências absolutas (№) e relativas (\%), quando se tratou de variável categórica e pela leitura das medidas de posição - média, variabilidade e desvio padrão, quando a variável foi quantitativa.

O teste qui - quadrado testou a hipótese de associação com nível de significância de $5 \%(P<0,05)$. Os resultados foram apresentados na forma de tabela.

O início da coleta de dados deu-se após a aprovação do projeto de pesquisa pelo Comitê de Ética em Pesquisa - CEP do Centro Universitário UNINOVAFAPI e pelo Comitê de Ética e Pesquisa da instituição coparticipante respeitando a Resolução 466/2012; a qual trata das diretrizes e normas de pesquisas envolvendo seres humanos, como por exemplo, a garantia do sigilo e a liberdade de recusa ou retirada do seu consentimento em qualquer fase do estudo.

\section{RESULTADOS}

Na primeira parte do questionário foi apresentada a distribuição dos participantes da pesquisa segundo as características sócio-demográficas e formação acadêmica, conforme a Tabela 1. Quanto ao quesito sexo, observou-se maior frequência de mulheres $-96,15 \%$ do total pesquisado. O sexo masculino representou $3,85 \%$ da amostra.

Com relação à idade, a maior parte dos profissionais, 57,70\% estava na faixa entre 26 a 35 anos, 19,23\% entre 36 a 45 anos, $15,38 \%$ na faixa etária de 46 a 55 anos e $7,69 \%$ até 25 anos. No tocante ao estado civil observou-se que $50 \%$ são casados, $46,15 \%$ solteiros, $3,85 \%$ divorciados. Referente à formação escolar, $50 \%$ tinham $2^{\circ}$ grau completo e $19,23 \%$ nível superior completo, $11,54 \%$ superior incompleto, $11,54 \%$ tinham pósgraduação e $7,69 \% 2^{\circ}$ grau incompleto. Em relação a formação $88,46 \%$ são técnicos de enfermagem e $11,54 \%$ são enfermeiros.

Quanto ao tempo de exercício da profissão, $50 \%$ dos profissionais estão entre um a cinco anos, 30,77\% tem de seis a dez anos, 7,69\% tem entre 11 a 15 anos e $7,69 \%$ tem de 16 ou mais anos e $3,85 \%$ menos de um ano. Com relação ao tempo de exercício da profissão no CC nota-se que $46,15 \%$ dos profissionais estão entre um a cinco anos, 30,77\% tem de seis a dez anos, $11,54 \%$ tem menos de um ano, $7,69 \%$ tem de 16 ou mais anos e 3,85\% entre 11 a 15 anos. Quanto ao tempo de trabalho na instituição, $61,53 \%$ dos profissionais estão entre um a cinco anos, $23,07 \%$ tem de seis a dez anos, $7,69 \%$ tem menos de um ano, 3,84\% tem entre 11 a 15 anos e $3,84 \%$ de 16 ou mais anos.

As questões com menor acerto pelos participantes foram as de números 3,4 e 9 , sendo que a questão de número 9 só obteve $34,61 \%$ de acertos e as questões de número 3 e 4 tiveram uma margem de $42,31 \%$. A questão com menor acerto pelos participantes foi referente a áreas altamente contaminadas próximas ao sítio cirúrgico (ânus, colostomia) que devem ser isoladas com adesivo estéril. 
Tabela 1 - Perfil Sociodemográfico e Formação Acadêmica, n= 08. Teresina-PI, 2018.

\begin{tabular}{|c|c|c|}
\hline $\begin{array}{l}\text { Perfil Sociodemográfico e } \\
\text { Formação Acadêmica }\end{array}$ & № & $\%$ \\
\hline $\begin{array}{l}\text { Sexo } \\
\text { Masculino } \\
\text { Femenino } \\
\text { Total } \\
\end{array}$ & $\begin{array}{c}1 \\
25 \\
26 \\
\end{array}$ & $\begin{array}{c}3,85 \\
96,15 \\
100 \\
\end{array}$ \\
\hline $\begin{array}{l}\text { Faixa Etária (anos) } \\
\text { Até } 25 \\
26 \text { a } 35 \\
36 \text { a } 45 \\
46 \text { a } 55 \\
56 \text { ou mais } \\
\text { Total }\end{array}$ & $\begin{array}{c}2 \\
15 \\
5 \\
4 \\
- \\
26\end{array}$ & $\begin{array}{c}7,69 \\
57,69 \\
19,23 \\
15,38 \\
- \\
100\end{array}$ \\
\hline $\begin{array}{l}\text { Estado Civil } \\
\text { Solteiro } \\
\text { Casado } \\
\text { União Estável } \\
\text { Divorciado, desquitado } \\
\text { Viúvo } \\
\text { Total } \\
\end{array}$ & $\begin{array}{c}12 \\
13 \\
- \\
1 \\
- \\
26\end{array}$ & $\begin{array}{l}46,15 \\
50 \\
- \\
3,85 \\
- \\
100\end{array}$ \\
\hline $\begin{array}{l}\text { Escolaridade } \\
1^{\circ} \text { grau completo } \\
2^{\circ} \text { grau incompleto } \\
2^{\circ} \text { grau completo } \\
\text { Superior incompleto } \\
\text { Superior completo } \\
\text { Pós-graduação } \\
\text { Total }\end{array}$ & $\begin{array}{c}- \\
2 \\
13 \\
3 \\
5 \\
3 \\
26\end{array}$ & $\begin{array}{c}- \\
7,69 \\
50 \\
11,54 \\
19,23 \\
11,54 \\
100\end{array}$ \\
\hline $\begin{array}{l}\text { Formação } \\
\text { Enfermeiro } \\
\text { Técnico } \\
\text { Auxiliar } \\
\text { Total }\end{array}$ & $\begin{array}{c}3 \\
23 \\
- \\
26\end{array}$ & $\begin{array}{c}11,54 \\
88,46 \\
- \\
100\end{array}$ \\
\hline $\begin{array}{l}\text { Tempo de profissão (anos) } \\
\text { Menos de } 1 \\
1 \text { a } 5 \\
6 \text { a } 10 \\
11 \text { a } 15 \\
16 \text { ou mais } \\
\text { Total }\end{array}$ & $\begin{array}{c}1 \\
13 \\
8 \\
2 \\
2 \\
26\end{array}$ & $\begin{array}{c}3,85 \\
50 \\
30,77 \\
7,69 \\
7,69 \\
100\end{array}$ \\
\hline $\begin{array}{l}\text { Tempo de exercício da pro } \\
\text { Menos de } 1 \\
1 \text { a } 5 \\
6 \text { a } 10 \\
11 \text { a } 15 \\
16 \text { ou mais } \\
\text { Total }\end{array}$ & $\begin{array}{c}3 \\
3 \\
12 \\
8 \\
1 \\
2 \\
26 \\
\end{array}$ & $\begin{array}{c}11,54 \\
46,15 \\
30,77 \\
3,85 \\
7,69 \\
100 \\
\end{array}$ \\
\hline $\begin{array}{l}\text { Tempo trabalho na institui } \\
\text { Menos de } 1 \\
1 \text { a } 5 \\
6 \text { a } 10 \\
11 \text { a } 15 \\
16 \text { ou mais } \\
\text { Total }\end{array}$ & $\begin{array}{c}2 \\
16 \\
6 \\
1 \\
1 \\
26\end{array}$ & $\begin{array}{c}7,69 \\
61,54 \\
23,08 \\
3,85 \\
3,85 \\
100 \\
\end{array}$ \\
\hline
\end{tabular}

Fonte: Pesquisa direta.

Na Tabela 2 é apresentado a distribuição dos índices de acertos dos participantes no teste de conhecimento da equipe sobre os cuidados no pré e intraoperatório. Nota-se que dentre os 11 itens do teste, em três itens (7, 8 e 10) os participantes obtiveram $92 \%$ ou mais de acertos. Em cinco itens (1, 2, 5, 6 e 11) o índice de acertos foi maior que $73 \%$ e menor que $89 \%$. 
Tabela 2 - Conhecimento da equipe sobre cuidados com o paciente no pré e intraoperatório, n=06. Teresina-PI, 2018.

\section{Cuidados no pré e intraoperatório}

№

$22 \quad 84,61$

Indiferente

Não

Não sei

Total

2. O paciente deve ser crientado a não aplicar soluções à base de álcool, loções, emolientes ou cosméticos na pele após a real Sim Indiferente

Não

Não sei

Total

3. A tricotomia deve ser realizada somente em situações clínicas específicas. (V)

$$
\text { Sim }
$$

Indiferente

Não

Não sei

Total

4. Recomenda-se a manutenção dos pelos em pacientes submetidos a qualquer tipo de procedimento cirúrgico e, se absolutamente necese
Sim

Não

Não sei

Total

5. A tricotomia deve ser realizada em um período de tempo mais próximo possível da incisão cirúrgica em um ambiente externo

Sim
ndiferente
Não
Não sei
Total

6. Para realização da antissepsia da pele de pacientes que serão submetidos a procedimentos cirúrgicos, recomenda-se o empreg à base de álcool. (V)
84,61

3,85

311,54

100,00

banho pré-operatório. ( $\mathrm{V})$

$2 \quad 80,77 \quad 7,69$

$3 \quad 11,54$

$26 \quad 100,00$

$11 \quad 42,31$

$2 \quad 7,69$

$13 \quad 50,00$

$26 \quad 100,00$

cessária, com tricotomizador elétrico. (V)

$11 \quad 42,31$

$9 \quad 34,61$

$\begin{array}{ll}9 & 34,61 \\ 5 & 19,23\end{array}$

$26 \quad 100,00$

à sala operatória. (V)

1973,08

7,69

19,23

$26 \quad 100,00$

3,85

go de solução de gluconato de clorexidina Sim Indiferente

Não

Total

22

2

2

26

84,62

REAS/EJCH | Vol.Sup.31 | e1100 | DOI: https://doi.org/10.25248/reas.e1100.2019 Página 5 de 7 


\begin{tabular}{|c|c|c|c|}
\hline \multicolumn{4}{|c|}{ 7. O sítio cirúrgico deve estar livre de óleos, cosméticos, produtos à base de álcool e sujidade aparente. (V) } \\
\hline Sim & 24 & 92,30 & \\
\hline Indiferente & - & & - \\
\hline Não & 1 & & 3,85 \\
\hline Não sei & 1 & & 3,85 \\
\hline Total & 26 & 100,00 & \\
\hline \multicolumn{4}{|c|}{ 8. Realizar degermação da pele correspondente ao sítio cirúrgico antes da antissepsia. } \\
\hline Sim & 24 & 92,30 & \\
\hline Indiferente & - & & - \\
\hline Não & 1 & & 3,85 \\
\hline Não sei & 1 & & 3,85 \\
\hline Total & 26 & 100,00 & \\
\hline Sim & 24 & 92,30 & \\
\hline \multicolumn{4}{|c|}{ 9. Áreas altamente contaminadas próximas ao sítio cirúrgico (ex. ânus, colostomia) devem ser isoladas com adesivo estéril. (V) } \\
\hline Sim & 9 & 34,61 & \\
\hline Indiferente & 2 & & 7,69 \\
\hline Não & 11 & 42,32 & \\
\hline Não sei & 4 & 15,38 & \\
\hline Total & 26 & 100,00 & \\
\hline \multicolumn{4}{|l|}{ 10. Realizar antissepsia do sítio cirúrgico utilizando técnica asséptica. (V) } \\
\hline Sim & 25 & 96,15 & \\
\hline Indiferente & 1 & & 3,85 \\
\hline Não & - & & - \\
\hline Não sei & - & & - \\
\hline $\begin{array}{ll}\text { Total } \\
\end{array}$ & 26 & 100,00 & \\
\hline \multicolumn{4}{|c|}{$\begin{array}{l}\text { 11. Realizar preparo com degermação das áreas de maior contaminação, como: umbigo, glande, sob as unhas e estoma intestinal ou urinário quando fazem parte do campo } \\
\text { cirúraico. (V) }\end{array}$} \\
\hline Sim & 23 & 88,46 & \\
\hline Indiferente & 2 & & 7,69 \\
\hline Não & 1 & & 3,85 \\
\hline Não sei & - & & - \\
\hline Total & 26 & 100,00 & \\
\hline
\end{tabular}

Fonte: Pesquisa direta. 


\section{DISCUSSÃO}

Com base nos estudos realizados foi possível acrescentar algumas informações importantes relacionadas às Infecções de Sítio Cirúrgico, podendo entender que fatores que contribuem para Infecção de Sítio Cirúrgico podem ser evitados se houver cuidados adequados, como uma correta degermação das mãos, segurança na esterilidade dos materiais, atendimento eficaz no pré-operatório, no intraoperatório e no pós-operatório. Neste estudo notou-se que a equipe de enfermagem é predominantemente feminina (96,15\%), a presença masculina representou apenas 3,85\%. Historicamente, a Enfermagem sempre foi considerada uma profissão de caráter feminino; atualmente esse quadro está se modificando, ainda que seja um processo lento já se pode notar a presença masculina em toda área voltada para a Enfermagem (SOUZA LL, 2014).

Nota-se neste estudo que $50 \%$ dos profissionais tem de um a cinco anos de atuação na profissão de Enfermagem. Esse parâmetro pode ser relacionado com outros itens, como tempo de exercício de profissão e tempo de trabalho na instituição, os quais também tiveram como maioria o resultado de um a cinco anos. Logo, ao analisar estes três itens pode-se notar que mais da metade da equipe começou a sua carreira profissional nesse hospital em estudo. Também se observa que esse pouco tempo de serviço explica a parte deficitária nas respostas sobre conhecimento destes profissionais a respeito de alguns aspectos da prevenção de Infecção de Sítio Cirúrgico (KUNZLE SRM, PEREIRA CS, ALVES KC, PELÁ NTR et al., 2006).

A Tabela 2 apresenta que os enfermeiros e os técnicos de Enfermagem possuíam domínio de grande parte dos assuntos referente ao cuidado com o paciente no pré e no intra-operatório, assuntos como banho pré-operatório e orientações ao paciente sobre evitar loções após o banho pré-operatório, juntamente com a importância da realização da antissepsia da pele e sobre a realização correta da degermação das áreas de maior contaminação. São assuntos de fundamental importância para uma boa prevenção de ISC, pois o banho pré-operatório deve ser feito com um produto antisséptico para a lavagem da pele, pois esse procedimento diminui consideravelmente as bactérias desta. Isso faz com que haja redução da microflora da pele, levando a menor incidência de ISC, o que leva a uma menor incidência de ISC. O paciente tem que ser orientado a não fazer uso de nenhum produto como loções a base de álcool ou cosméticos na pele após 0 banho (WEBSTER J; OSBORNE S, 2015).

\section{CONCLUSÃO}

Apesar do bom nível de conhecimento demonstrado pela população deste estudo, infere-se que há necessidade de estratégias de aperfeiçoamento e atualização no que diz respeito a prevenção de infecção de sítio cirúrgico. O déficit de conhecimento acerca deste tema poderá ser apresentado e minimizado por meio de treinamento a ser oferecido ao grupo de profissionais deste serviço pelos pesquisadores, como contribuição deste estudo. É essencial que sejam continuamente promovidas essas medidas de aperfeiçoamento aos profissionais, pois se trata de um tema que está constantemente se modernizando.

\section{REFERÊNCIAS}

1. ANVISA. Segurança do Paciente e Qualidade em Serviços de Saúde: Critérios Diagnósticos de Infecção Relacionada à Assistência à Saúde. 1ª edição. Brasília, 2013.

2. GIL, A. C. Como elaborar projetos de pesquisa. 5. ed. São Paulo: Atlas, 2008.

3. KUNZLE SRM, PEREIRA CS, ALVES KC, PELÁ NTR, GIRE. Auxiliares e Técnicos de Enfermagem e controle de infecção hospitalar em centro cirúrgico: mitos e verdades. Revista Esc. Enf. USP, 2006; 40(2): 214-20.

4. MARCONI MA, LAKATOS EM. Fundamentos de Metodologia Científica. 7 ed. São Paulo, 2010.

5. PADOVEZE MC, FORTALEZA CMCB. Infecções relacionadas à assistência à saúde: desafios para a saúde pública no Brasil. Revista de Saúde Pública, 2014; 48 (6).

6. REIS RG, RODRIGUES MCS. Infecção De Sítio Cirúrgico Pós-Alta: ocorrência e caracterização de egressos de cirurgia geral. Cogitare Enferm, 2017; 22 (4).

7. SOBECC. Práticas Recomendadas SOBECC - Centro de Material e Esterilização, Centro Cirúrgico, Recuperação Pós-Anestésica. $7^{\text {a }}$ ed. São Paulo: SOBECC Associação, 2017.

8. SOUZA LL. Representações de gênero na prática de enfermagem na perspectiva de estudantes. Revista Ciências \& Cognição, $2014 ; 19(2): 218-232$.

9. WEBSTER J, OSBORNE S. Preoperative bathing or showering with skin antiseptics to prevent surgical site infection. Cochrane. Revista online: Wounds Group: 19 de fevereiro de 2015.

10. ZANELLA C. Metodologia de estudo de pesquisa em administração. Fascículo de Metodologia Científica, UFSC/MEC/CAPES/PNAP, Florianópolis, 2009. 